$1-20-2003$

\title{
Experimental Observation of Total-Internal-Reflection Rainbows
}

Charles L. Adler

James A. Lock

Cleveland State University, j.lock@csuohio.edu

Jonathon Mulholland

Brian Keating

Diana Ekelman

Follow this and additional works at: https://engagedscholarship.csuohio.edu/sciphysics_facpub

Part of the Physics Commons

How does access to this work benefit you? Let us know!

\section{Publisher's Statement}

This paper was published in Applied Optics and is made available as an electronic reprint with the permission of OSA. The paper can be found at the following URL on the OSA website: http://www.opticsinfobase.org/ao/abstract.cfm?URI=ao-42-3-406. Systematic or multiple reproduction or distribution to multiple locations via electronic or other means is prohibited and is subject to penalties under law.

\section{Original Citation}

Adler, Charles L., James A. Lock, Jonathon Mulholland, Brian Keating, and Diana Ekelman. "Experimental Observation of Total-Internal-Reflection Rainbows." Applied Optics 42 (2003): 406-411.

\section{Repository Citation}

Adler, Charles L.; Lock, James A.; Mulholland, Jonathon; Keating, Brian; and Ekelman, Diana, "Experimental Observation of Total-Internal-Reflection Rainbows" (2003). Physics Faculty Publications. 43.

https://engagedscholarship.csuohio.edu/sciphysics_facpub/43

This Article is brought to you for free and open access by the Physics Department at EngagedScholarship@CSU. It has been accepted for inclusion in Physics Faculty Publications by an authorized administrator of EngagedScholarship@CSU. For more information, please contact library.es@csuohio.edu. 


\title{
Experimental observation of total-internal-reflection rainbows
}

\author{
Charles L. Adler, James A. Lock, Jonathon Mulholland, Brian Keating, and Diana Ekelman
}

\begin{abstract}
A new class of rainbows is created when a droplet is illuminated from the inside by a point light source. The position of the rainbow depends on both the index of refraction of the droplet and the position of the light source, and the rainbow vanishes when the point source is too close to the center of the droplet. Here we experimentally measure the position of the transmission and one-internal-reflection totalinternal-reflection rainbows, and the standard (primary) rainbow, as a function of light-source position. (C) 2003 Optical Society of America

OCIS codes: $\quad 010.1290,290.1310,290.5850$.
\end{abstract}

\section{Introduction}

In the standard geometrical optics theory of the rainbow, the position of the rainbow is derived by assuming that a bundle of parallel light rays are refracted into a water droplet, make a number of reflections inside the droplet, and refract out. The rainbow occurs at a relative minimum in the deflection angle as a function of the impact parameter of light rays entering the droplet. The assumption that the rays are parallel means that the light source illuminating the droplet is infinitely far away. (Realistic models of the natural rainbow also must take into account the finite angular width of the Sun, but we will ignore this complication. ${ }^{1}$ ) We can imagine taking a point source of light and moving it from a great distance away from the droplet, bringing it closer and closer, and finally inside the water droplet. Does the nature of the rainbow change when we bring the light source inside?

It does, in two ways:

(a) First, a fundamentally new class of rainbows is created [total-internal-reflection (TIR) rainbows

C. L. Adler (cladler@smcm.edu), J. Mulholland, B. Keating, and D. Ekelman are with the Department of Physics, St. Mary's College of Maryland, St. Mary's City, Maryland 20686. J. A. Lock is with the Department of Physics, Cleveland State University, Cleveland, Ohio 44115 .

Received 23 January 2002; revised manuscript received 12 April 2002.

0003-6935/03/030406-06 $\$ 15.00 / 0$

(C) 2003 Optical Society of America
(TIRRs)] when the light source is moved inside the droplet.

(b) Second, the standard rainbows and TIRRs do not exist for all positions of the light source. The rainbow angles are a function of the position of the light source and cease to exist when the light source is moved too close to the center of the droplet.

\section{Theory}

A semiclassical treatment of the scattering of light by an internal light source in a spherical droplet has been derived recently. ${ }^{2}$ In this paper we look only at what geometrical optics can tell us about this problem. Figure 1 shows a diagram of a droplet of radius $a$ with a point source of light inside it at a distance $r$ from the center. The index of refraction of the droplet is $n$, whereas the index of the surrounding medium is $1 . \mathrm{A}$ light ray from the source is internally reflected $p-1$ times before leaving the droplet. We define several angles as follows: $\tau$ is the angle that the light ray makes with respect to a diameter of the droplet that runs through the light source, $\alpha$ is the angle that the ray makes with the surface normal to the droplet as it exits the droplet, $\gamma$ is the angle between the ray and the surface normal when the ray is refracted out of the droplet, and $\theta$ is the total deflection angle from $0^{\circ}$. From Snell's law and the law of reflection, we can derive several relationships among these angles:

$$
\begin{aligned}
(r / a) \sin \tau & =\sin \alpha, \\
n \sin \alpha & =\sin \gamma, \\
\theta & =\tau+(p-1) \pi-(2 p-1) \alpha+\gamma .
\end{aligned}
$$




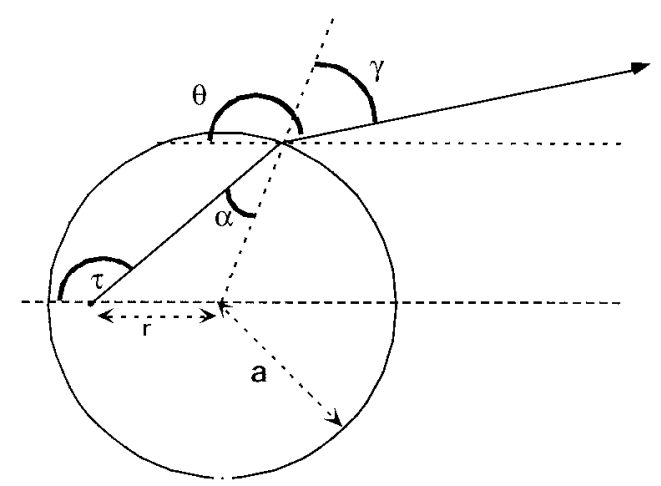

Fig. 1. Ray diagram for light scattered by a point light source inside a spherical droplet.

Because $\theta$ can be larger than $180^{\circ}$, we define the scattering angle, $\phi$, to be $\theta$ reduced to the range $0-180^{\circ}$. Because the light source is inside the droplet, for certain values of $r / a$ and $\tau$, light will be totally internally reflected inside the droplet. Figure 2 shows $\phi$ as a function of $\tau$ for $r / a=0.8, n=4 / 3$, and $p=1$ (i.e., no internal reflections). For $\tau$ between approximately $80^{\circ}$ and $110^{\circ}$, the light is totally internally reflected. What is interesting is that for values of $\tau$ just beyond the TIR region, $\phi$ goes through a shallow minimum for a scattering angle that is otherwise forbidden because of TIR. This minimum angle represents a scattering angle for which the intensity of light (from geometrical optics) diverges: in other words, a rainbow.

By considering the conditions under which $d \theta / d \tau=$ 0 , we can examine what range of values of $\zeta=r / a$ for which a TIRR can occur. From Eqs. (1)-(3),

$$
\frac{\mathrm{d} \theta}{\mathrm{d} \tau}=1-(2 p-1) \xi \frac{\cos \tau}{\cos \alpha}+n \xi \frac{\cos \tau}{\cos \gamma}
$$

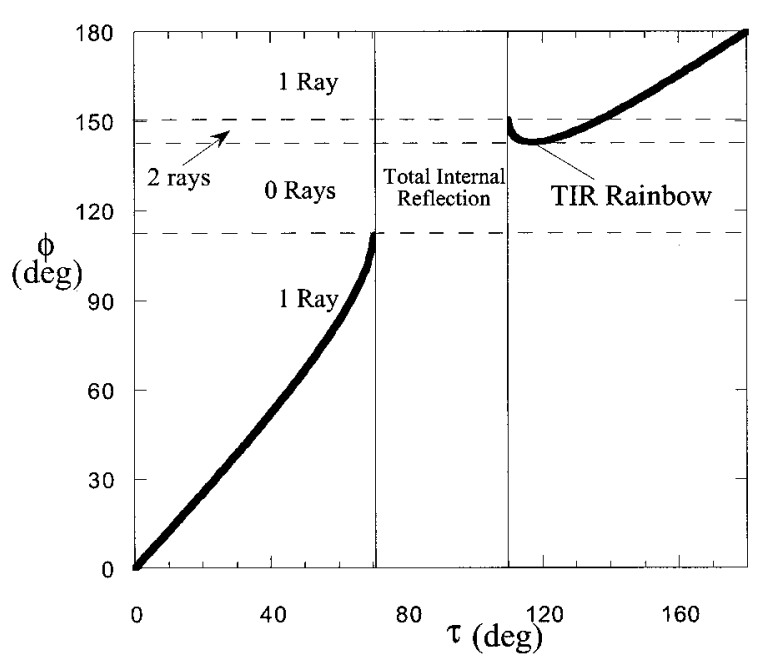

Fig. 2. Scattering angle $(\theta)$ as a function of the internal impact parameter $(\tau)$ for zero internal reflections $(p=1)$. For this diagram, $r / a=0.8$ and $n=1.33$.

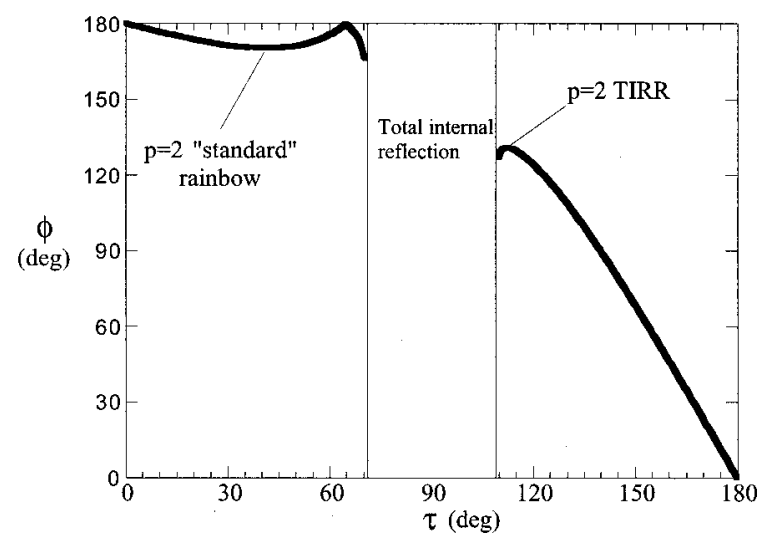

Fig. 3. Scattering angle $(\theta)$ as a function of internal impact parameter $(\tau)$ for one internal reflection $(p=2)$. For this diagram, $r / a=0.8$ and $n=1.33$.

Setting the derivative to 0, and using Eqs. (1)-(3) to substitute for $\cos \alpha$ and $\cos \gamma$, we find

$$
\begin{aligned}
& 1+\frac{n \xi x}{\left[1-n^{2} \xi^{2}\left(1-x^{2}\right)\right]^{1 / 2}}-(2 p-1) \\
& \times \frac{\xi x}{\left[1-\xi^{2}\left(1-x^{2}\right)\right]^{1 / 2}}=0,
\end{aligned}
$$

where $x=\cos \tau$ in this expression. Since $x \leq 1, \xi \geq$ $1 / n$ if there is to be a zero for this expression; thus this TIRR occurs for values of $r / a$ between 0.75 and 1 for $n=4 / 3$. For this index the rainbow angle $\theta$ varies between $131^{\circ}$ (for $r / a=0.75$ ) and $158.5^{\circ}$ (for $r / a=1)$.

If light is reflected once before exiting the water droplet, potentially two different types of rainbow can be created: the standard $p=2$ rainbow, and, because some of the rays from the point source will be totally internally reflected, a $p=2$ TIRR. Figure 3 is a graph of $\theta$ versus $\tau$ for the $p=2$ case for $r / a=0.8$ and $n=4 / 3$. The position of the $p=2$ standard rainbow and the $p=2$ TIRR are indicated on the graph. (The dent in the graph near $\tau=66^{\circ}$ is an artifact caused by $\theta$ exceeding $180^{\circ}$ at that point.) The behavior of the $p=2$ standard rainbow as the position of the light source is varied is interesting. For $r / a<0.6$, the $p=2$ rainbow does not occur; we can show this by setting $x=1$ in Eq. (5) and solving for $\xi$. For $r / a=0.6$ the rainbow angle is $180^{\circ}$, which is essentially rainbowenhanced glory scattering. ${ }^{3,4}$ As $r / a$ approaches 1 , the $p=2$ standard rainbow and the $p=1$ TIRR merge; this is because a point source located near the edge of the droplet will have the TIR region located almost directly behind it on the nearer edge of the droplet. ${ }^{2}$ In principle, TIRRs can exist for all values of $p$, and the standard rainbow can exist for all $p \geq 2$, but we do not consider them in this paper, because they become faint; experimentally, we did not see any of the $p>2$ rainbows. 


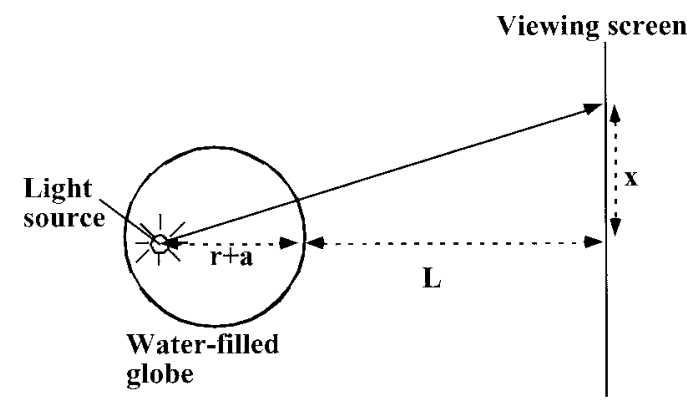

Fig. 4. Experimental setup. In our experiment, $L=67.5 \mathrm{~cm}$ and $a=8 \mathrm{~cm}$.

\section{Experiment}

Figure 4 shows a diagram of the experimental setup we used to investigate TIRRs. We used a waterfilled globe (inner diameter, $16 \mathrm{~cm}$; wall thickness, $0.13 \mathrm{~cm}$ ) to simulate a water droplet and placed a point source of light inside the droplet. Light exiting the droplet was projected onto a white screen. The positions of the $p=1$ and $p=2$ TIRRs and the $p=$ 2 standard rainbow were measured on the screen with a meter stick; in addition to this, we photographed the rainbows, using a high-resolution digital camera. The point light source that we used was a bulb from a mini-Maglite flashlight; to hold the bulb in place, we pushed wires through a double-barreled plastic coffee stirrer, looped them around the end prongs of the bulb, and pulled the end of the bulb into the coffee stirrer. This gave us an electrical contact of such quality that we did not have to solder the leads to the wire. We then wrapped the end of the tube with waterproof tape to seal the bulb from water. The size of the filament is approximately $1 \mathrm{~mm}$ and is located $3 \mathrm{~mm}$ from the end of the bulb. Figure 5 is a photo of the experimental setup; the bulb was held on a mounting arm that was in turn supported on a translation stage used to change the position of the light source. We used a low-voltage power sup-

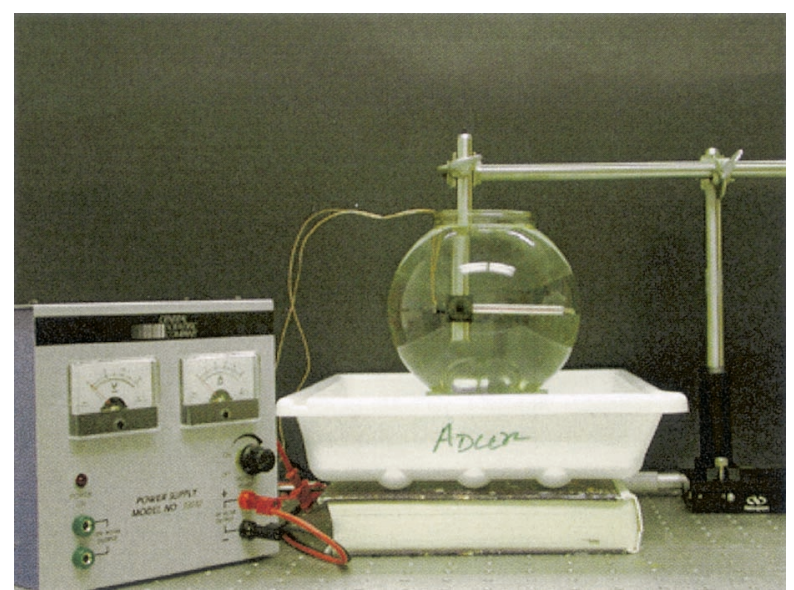

Fig. 5. Photograph of droplet and assembly used to move the light source. The cross arm holding the light bulb was built on a linear translation stage, which was moved in $0.5-\mathrm{mm}$ increments to change the position of the bulb. ply to illuminate the bulb; experimentally, we found that the bulb could be powered with a voltage of $2.5 \mathrm{~V}$ without burning it out. The height of the bulb was 8 $\mathrm{cm}$ above the bottom of the globe.

Figure 6 shows photographs of the rainbows from the water-filled globe projected onto a white screen at a distance of $L=67.5 \mathrm{~cm}$ behind the globe. The $p=$ 1 TIRR, the $p=2$ standard rainbow(s), and the $p=$ 2 TIRR are labeled on the figures. The sequence of Fig. 6 shows a monotonic decrease in $r / a$ from 0.96 (which is as close to the wall of the globe as we can get our light source) down to $r / a=0.6$, where the $p=2$ standard rainbow ceases to exist. It is seen that at $r / a=0.75$, the $p=1$ and $p=2$ TIRRs vanish as predicted. Note that they move in opposite directions and that their colors are also oriented in the opposite manner. At $r / a=0.6$, the $p=2$ standard rainbow scattering angle is $180^{\circ}$, and for values of $r / a$ less than this, it is gone.

Although it is not immediately obvious from the photograph, there are in fact two $p=2$ standard rainbows, which we label $\alpha$ and $\beta$. We see two $p=$ 2 standard rainbows because of the glass in the globe: Fig. 7 shows the two ray paths that contribute to the $\alpha$ and $\beta$ rainbows. Because the relative index difference between glass and air is greater than for water and glass, the $\beta$ rainbow will be brighter than the $\alpha$. The angular separation between the $\alpha$ and the $\beta$ rainbows is proportional to the glass thickness divided by the radius of the globe. ${ }^{5,6}$ Because there are two possible internal reflections for the $p=2$ TIRR (the internal reflection occuring at the waterglass or the glass-air interface), one might expect to see $2 p=2$ TIRRs; however, only one was seen. We conjecture that the second is too faint to be seen against the background of scattered light from the globe.

Figure 8 shows a graph of the scattering angle as a function of $r / a$ for the $p=1$ and $p=2$ TIRRs and the $p=2$ standard rainbow. Both experiment and theory are shown. For the theory, two corrections had to be made to the rainbow angle: One was due to the glass thickness, and one was due to a geometrical correction resulting from the fact that all experimental angles were measured with respect to the center of the glass globe and the scattering screen is not infinitely far away from the globe. The theory and experiment match well, except for the $p=2$ TIRR, where the theory and experiment are a few degrees apart but have the same functional dependence on $r / a$. We are not certain why the discrepancy is so large for the $p=2$ TIRR but conjecture that it is because the globe is not a perfect sphere and that departures from sphericity will have a larger effect on the positions of the higher-order rainbows than on the lower-order ones. ${ }^{7}$ Although we did not examine the colors of these rainbows in detail, from experiment the angular width of the rainbows was of the order of $1-2^{\circ}$ for all four rainbows for $r / a=0.9$. One can use Eqs. (1)-(5) and the dispersion of the refractive index of water to predict the angular width; for $r / a=0.9$, the angular width of the rainbow is pre- 


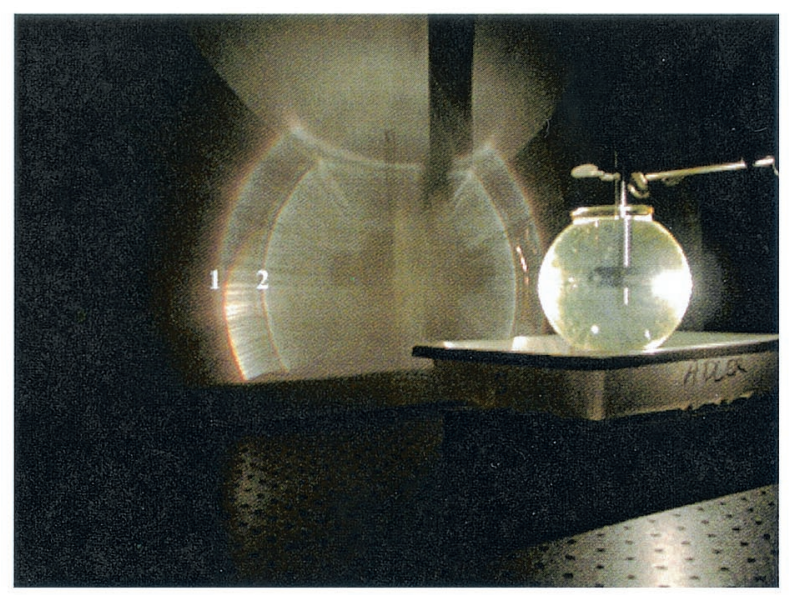

(a)

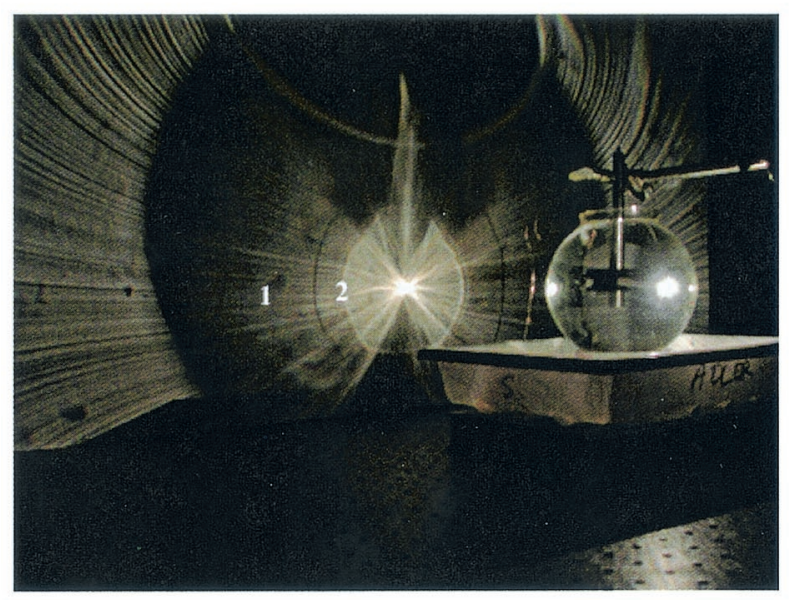

(c)

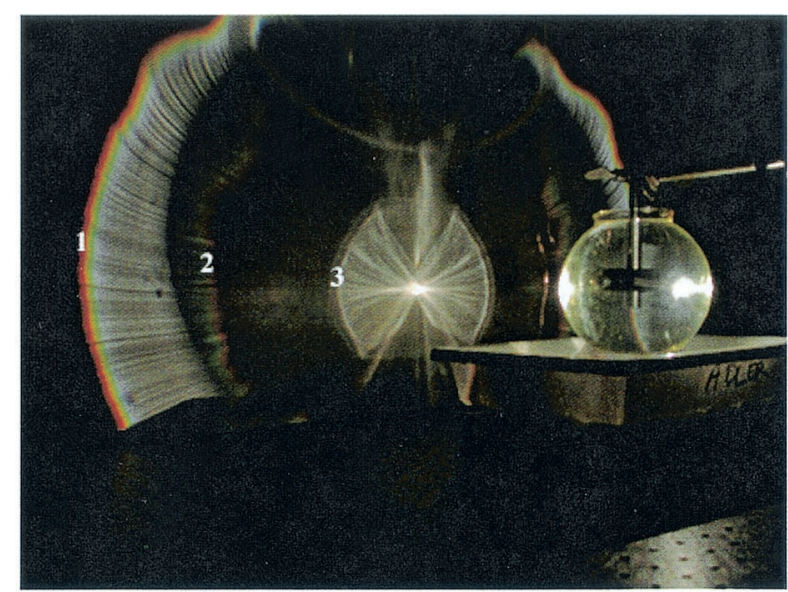

(b)

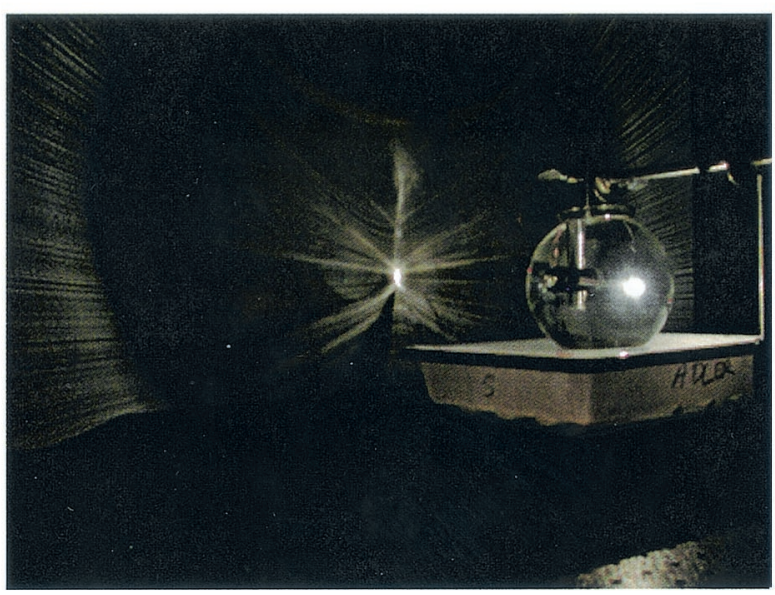

(d)

Fig. 6. Photographs of the light scattered by the droplet projected onto a screen. (a) $r / a=0.96 .1 . p=1$ TIRR rainbow, $2 . p=2$ standard rainbow. (b) $r / a=0.8 .1 . p=1$ TIRR rainbow, $2 . p=2$ TIRR rainbow, 3. $p=2$ "standard" rainbow. (c) $r / a=0.75$. At this light source position, the $p=1$ TIRR vanishes, leaving only the $p=2$ TIRR and the standard rainbows. 1. $p=2$ TIRR rainbow, 2 . $p=2$ standard rainbow. (d) $r / a=0.6$. At this light source position, the $p=2$ standard rainbows converge at a scattering angle of $\theta=180^{\circ}$. For smaller values of $r / a$, they cease to exist.

dicted to be $0.71^{\circ}$ for the $p=1$ TIRR, $1.56^{\circ}$ for the $p=$ 2 TIRR, and $0.46^{\circ}$ for the $p=2$ standard rainbow. This is in decent agreement with observation, although it does not take into account the refractive effects of the glass or its dispersion.

If we walk around the globe and look at the light

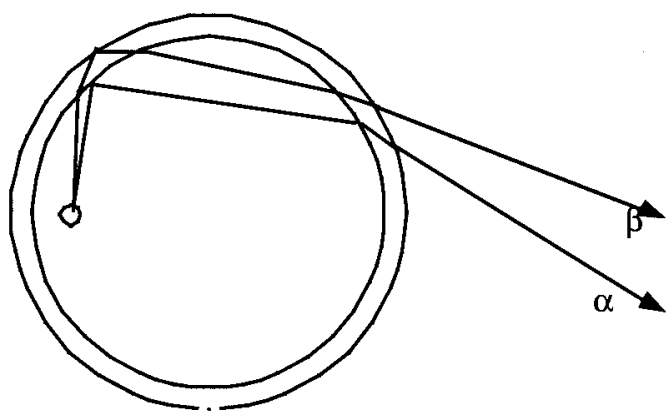

Fig. 7. Ray diagram showing the origin of the $\alpha$ and $\beta$ rainbows.

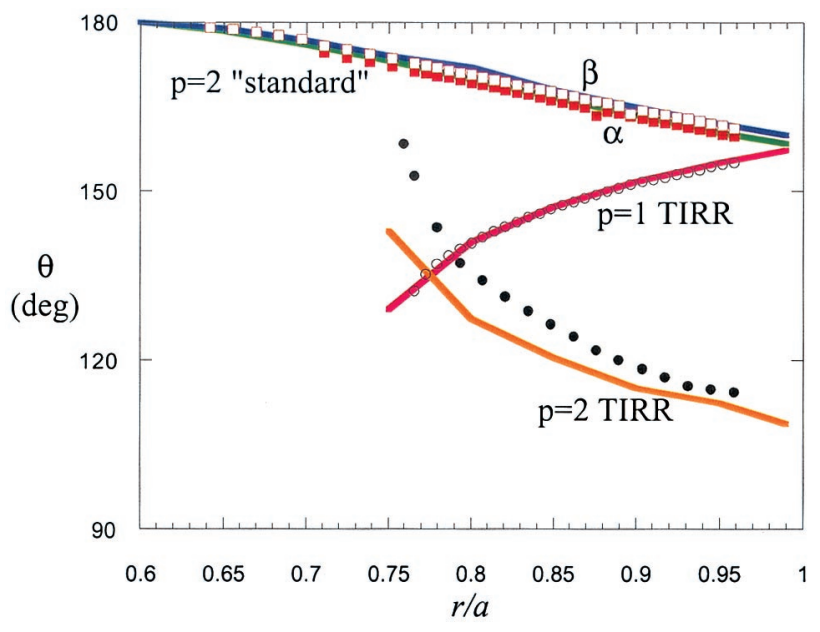

Fig. 8. Position of the $p=1$ and $p=2$ TIRRs and the $p=2$ standard $\alpha$ and $\beta$ rainbows as a function of light source position. Points, experiment; solid curve, theoretical fit. 


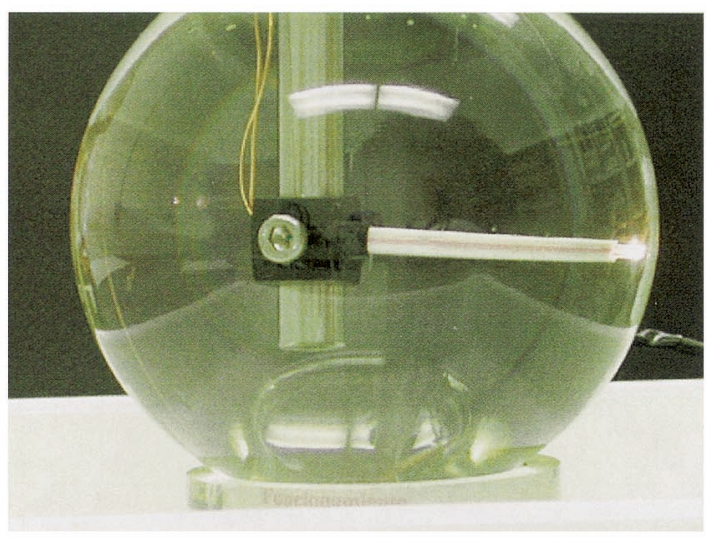

(a)

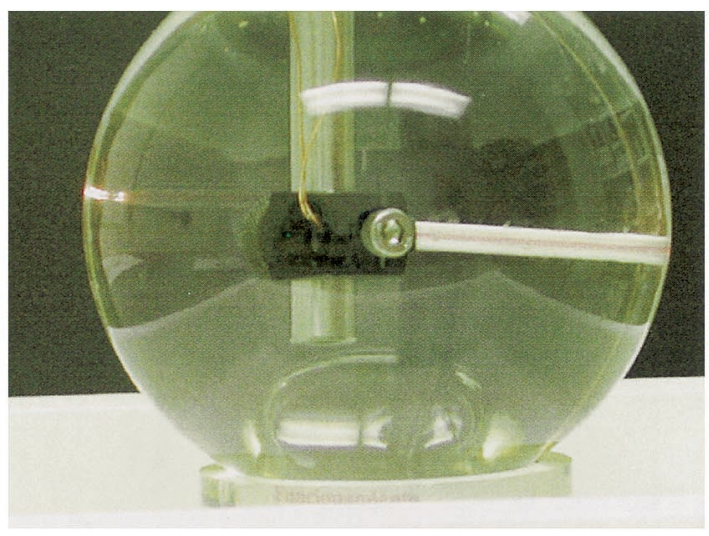

(b)

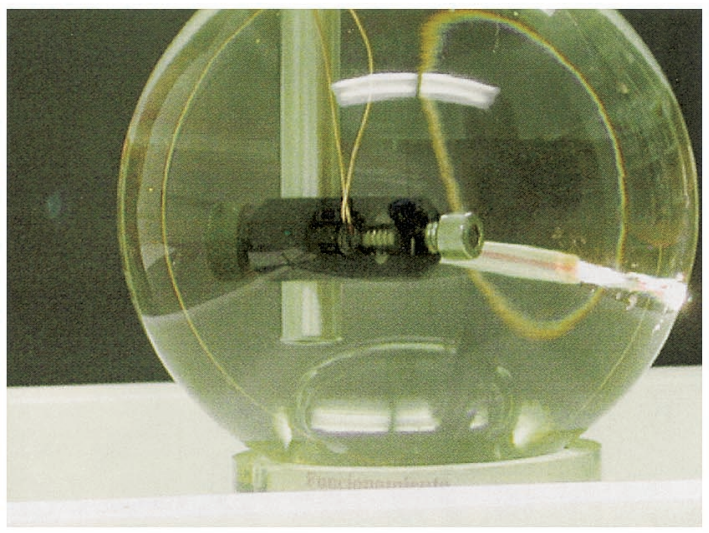

(c)

Fig. 9. Glare-spot sequence. $r / a=0.9$. (a) One-ray region. Light source clearly visible; one image of it seen. (b) Zero-ray region. Light source invisible because of total internal reflection. (c) Two-ray region. Two images of light source visible. The light source is in about the right position for the viewer to see the $p=$ 1 TIRR.

source, we will see some interesting things as we change our viewpoint. Figure 9 shows a glare-spot sequence for $r / a \sim 0.9$. In this sequence we are looking directly at the globe while changing the viewing angle of the observer. The position of the light source is kept constant. We start out at a viewing angle corresponding to a scattering angle near $90^{\circ}$ and increase the viewing angle through the sequence to the point where the observer's eye is in the region to intercept rays from the $p=1$ TIRR. In Fig. 9(a) one image of the light source is visible, corresponding to a one-ray region. In Fig. 9(b), when we increase the viewer's observing angle further, the light source cannot be seen because of TIR. In Fig. 9(c) two images of the light source are seen, corresponding to the $p=1$ TIRR.

\section{Conclusions}

By illuminating a water droplet from the inside, we have created and seen a fundamentally new class of rainbows that to our knowledge have never been recognized or closely examined before. One potential field where this research may be relevant is in the excitation of morphology-dependant resonances and the examination of single-molecule fluorescence in levitated droplets, droplet streams, and solid particles. ${ }^{8-11}$ There are intriguing data in the paper by Hill et al. ${ }^{11}$ in which they measured the angular dependence of the fluorescence of Coumarin dye molecules in a micodroplet. For three-photon excitation of the dye there seems to be an excess of fluorescence at angles near $150^{\circ}$. This may be significant, as the rate of three-photon excitation is proportional to the product of the intensity of three laser fields inside the droplet; because of this, the region where the probability of three-photon excitation of the dye is high is localized. Because the fluorescence is coming from a localized region inside the droplet, the excess signal is possibly an example of a $p=1$ TIRR. ${ }^{11}$ We are currently putting together an experiment to measure the intensity of the rainbow as a function of scattering angle, to allow further comparison with singlemolecule fluorescence results.

As far as examples from meteorological optics go, Young ${ }^{12}$ made an interesting observation during the 2001 Meteorological Optics Conference: Consider reversing the direction of light rays so that we have rays from outside the sphere converging on a point inside it. If we place an observer at this point, and consider the bowl to be the observer's sky, then the observer will see multiple images of parallel rays that hit the sky at angles near the TIRR impact parameters. This is analagous to the refraction of light rays in a stratified atmosphere, which produces multiple, distorted images of the setting Sun. ${ }^{12}$

We thank our two reviewers for a number of suggestions that strengthened the paper, especially the reviewer who called our attention to Refs. 9-11. This research was supported by National Science Foundation (NSF) grant PHY-9987862 and by NASA grant NCC3-521.

\section{References}

1. R. L. Lee, Jr., "What are 'all the colors of the rainbow'? Appl. Opt. 30, 3401-3407 (1991).

2. J. A. Lock, "Semi-classical scattering of an electric dipole source inside a spherical particle," J. Opt. Soc. Am. A 18, 3085-3097 (2001).

3. H. M. Nussenzveig, "Complex angular momentum theory of 
the rainbow and the glory," J. Opt. Soc. Am. 69, 1068-1079 (1979).

4. D. S. Langley and M. J. Morrell, "Rainbow-enhanced forward and backward glory scattering," Appl. Opt. 30, 3459-3467 (1991).

5. J. A. Lock, J. M. Jamison, and C.-Y. Lin, "Rainbow scattering by a coated sphere," Appl. Opt. 33, 4677-4690 (1994).

6. C. L. Adler, J. A. Lock, J. K. Nash, and K. W. Saunders, "Experimental observation of rainbow scattering by a coated cylinder: twin primary rainbows and thin-film interference," Appl. Opt. 40, 1548-1558 (2001).

7. C. L. Adler, J. A. Lock, and B. R. Stone, "Rainbow scattering by a cylinder with a nearly elliptical cross section," Appl. Opt. 37, 1540-1550 (1998).

8. M. Kerker and S. D. Druger, "Raman and fluorescent scattering by molecules embedded in spheres with radii up to several multiples of the wavelength," Appl. Opt. 18, 11721179 (1979).

9. M. D. Barnes, K. C. Ng, W. B. Whitten, and J. M. Ramsey, "Detection of single Rhodamine 6G molecules in levitated microdroplets," Anal. Chem. 65, 2360 (1993).

10. M. D. Barnes, N. Lermer, C.-Y. Kung, W. B. Whitten, J. M. Ramsey, and S. C. Hill, "Real-time observation of singlemolecule fluorescence in microdroplet streams," Opt. Lett. 22, 1265-1267 (1997).

11. S. C. Hill, V. Boutou, J. Yu, S. Ramstein, J. P. Wolf, W. L. Pan, S. Holler, and R. K. Chang, "Enhanced backward-directed multiphoton-excited fluorescence from dielectric microcavities," Phys. Rev. Lett. 85, 54-57 (2000).

12. A. Young, "Green flashes," presented at the 7th Topical Meeting on Meteorological Optics, Boulder, Colo., 6-8 June 2001. 